\title{
A review on recent research studies on vibration analysis of fluid- conveying nanotubes
}

\author{
E. Fatahian ${ }^{*}$, E. Hosseini ${ }^{2}$, H. Fatahian ${ }^{1}$ \\ ${ }^{1}$ School of Aerospace Engineering, Universiti Sains Malaysia, 14300, Nibong Tebal, Penang, Malaysia \\ ${ }^{2}$ Department of Mechanical Engineering, Dezful Branch, Islamic Azad University, Dezful, Iran \\ *Email: esmaeelfatahian@gmail.com
}

\begin{abstract}
Nanotube (such as carbon and boron nitride nanotubes) is a key component of modern technology applications of nanostructures due to their unique mechanical, electrical, and physical characteristics such as high elasticity modulus, suitable heat transfer, and electrical conductivity. Carbon and boron nitride nanotubes are among the promising choices in nano-fluidic, gas storage, and drug delivery systems due to their hollow cylindrical shape and appropriate chemical, mechanical, and physical properties. Thermal vibration assessment should be conducted on fluid-conveying carbon nanotubes since the effect of thermal fluctuations on the mechanical characteristics of nanostructure are significant. Previous studies have revealed that when thermal vibration is taken into account, quantum effects can become extremely important in nanoscale electronics and structures. Hence, the present review focuses mostly on previous work on fluid-conveying nanotubes and the dynamical characteristics of size-dependent vibration and non-local strain gradient theory of fluid-conveying nanotubes. Furthermore, a special effort is made to address recent and rare investigations on the vibration of fluid-conveying nanotubes in thermal environment, as well as thermal vibration concerns of carbon nanotubes.
\end{abstract}

Indexed Terms- Nanotubes, Fluid-conveying, Vibration, Thermal environment.

\section{INTRODUCTION}

Nanoscience has developed specialized fields in materials, mechanical engineering [1-4], and thermal engineering [5, 6], construction, chemistry, biomedical, electronic equipment, biotechnology [7-9], and power generation, paving the way for the next industrial revolution. Because of their unique mechanical, electrical, and physical properties, nanotubes are an important component of modern nanotechnology applications. Carbon Nanotube (CNT) and graphene sheet are nanomaterials that reveal the enormous potential in the development of new sensors, gas detection, and composite material and have inspired a great deal of interest in the industry. The excellent mechanical, electronic, and thermal characteristics of these nanomaterials enable their application in a wide range of possibilities $[10,11]$, such as gas detection, solar panels, microbial identification, diagnosis systems, and composite material. Carbon nanotube, an essential allotrope of carbon, is composed of Single-Walled CNTs (SWCNTs) or MultiWalled CNTs (MWCNTs) [12, 13]. Carbon nanotubes are carbon allotropes having cylindrical nanostructures, which are the first group of nano-products [14]. The carbon nanotube is a honeycomblike structure formed of twisted graphite sheets. They are extraordinarily long and thin structures that are also robust, resistant, and adaptable [15]. If the carbon nanotube simply consists of a graphene pipe, it can be referred to as Single-Walled CNT, and if it consists of many graphene rolled layers, it is referred to as Multi-Walled CNT [16]. They can be utilized in a wide range of utilization in Micro/Nanoelectronic systems. Boron nitride is a chemically resilient refractory combination composed of nitrogen and boron atoms, which provides it with unique features including a high elasticity modulus and great heat transfer $[17,18]$. Boron Nitride Nanotubes (BNNTs) have superior piezoelectric characteristics to carbon nanotubes, makes it a preferred choice for nanoelectromechanical systems. 
Mechanical examination of micro/nanoelectromechanical systems is fast gaining interest as a research topic. The study of the impacts of fluid-solid interactions on mechanical response, for instance, dynamic response, is crucial for developing the best fluid-conveying nanostructures. Theories of continuum mechanics, such as classical and size-dependent theories, can be implemented to investigate micro/nanostructures. Size-dependent methods, such as non-local, strain gradient, and couple stress, are preferable and can yield findings that are more accurate. Researchers have been interested in the thermal vibration problems of the carbon nanotube, which may be employed as a nano-electronic component, and an atomic force microscope (AFM) tip. Many studies have been carried out on the use of strain gradient theory for dynamic studies of nanotubes. Thermal vibration assessment should be conducted on fluid-conveying carbon nanotube due to the considerable impact of thermal fluctuations on the mechanical properties of nanostructures.

Therefore, the current study, which is mostly a brief overview of fluid-conveying nanotubes, attempts to explain the dynamical behaviors of fluid-conveying nanotubes as well as their sizedependent vibration behavior and non-local strain gradient theory. Furthermore, the few and notable studies on the vibration of fluid-conveying nanotube in thermal environment, as well as thermal vibration problems of carbon nanotubes, have been reviewed and addressed in detail.

\section{FLUID-CONVEYING NANOTUBES}

Mechanical investigation of micro/nanoelectromechanical systems is a field of study that has recently attracted a lot of interest [19-21]. There is strong contact between solid and liquid parts in several potential nanoelectromechanical systems. Nanofluidics-based systems, for example, offer a wide range of utilization in several nanotechnology fields, including nano-medicine [22]. Considering the impact of fluid-solid interactions on mechanical response, such as dynamic response, is critical for an optimal design for fluid-conveying nanostructure because these nanostructures often work under applied loads. Carbon nanotubes and Boron Nitride nanotubes, for instance, have numerous outstanding properties, including a high elasticity modulus, strong heat transfer, and electrical conductivity, and other mechanical characteristics [7]. Fluid flow within carbon nanotubes is an important and complex research area [23-25]. The impact of internal moving fluid on the total mechanical behavior of carbon nanotube, on the other hand, is a major concern. The main purpose of fluid mechanics of flow inside carbon nanotubes is to investigate how the wall-fluid interaction and fluid viscosity influence velocity distribution, as well as how the velocity distribution varies with pressure gradient in a non-classical approach [26]. Many scientists are interested in investigating the dynamical behaviors of fluidconveying nanotubes [27] since they may be utilized in diverse nano-electromechanical systems such as fluid transfer and drug delivery [28]. They found that carbon nanotube is a tubular structure that can be utilized to transfer nano-flows after detecting them. Therefore, fluid-structure interactions might happen in fluid-conveying CNTs that may be examined similarly to macroscopic pipelines. For several decades, researchers have investigated fluid-conveying carbon nanotubes, and significant advancements have been accomplished. Wang [29] studied the impact of small length scales on doublewalled carbon fluid-conveying nanotube. Zhang et al. [30] analyzed the role of the surface on wave behaviors of SWCNTs. Lee and Chang [31] proposed a carbon nanotube embedded in an elastic medium and revealed that the nonlocal impact and influence of viscosity could modify the natural frequency of carbon nanotube. Mirramezani and Mirdamadi [32] investigated the influence of the Knudsen number on the fluid-conveying CNTs in terms of inner flow size. Furthermore, Zeighampour et al. [33] examined the effects of slip boundary conditions on double-walled CNTs, and they stated that increasing the Knudsen number reduced the stability of the double-walled fluid-conveying carbon nanotube. Cheng et al. [27] investigated the free vibration of a fluid-conveying nanotube consisting of carbon and boron nitride nanotubes. They evaluated how length ratio and non-local parameters affected the vibration behavior and stability of fluid-conveying nanotube. They revealed that the length ratio and the nonlocal parameter had a considerable impact on the nanotube's instability. Increases in either the length ratio or the nonlocal factor will make the system more unstable; the nonlocal parameter, in 
particular, has a substantial impact on the second-mode divergence. It can be observed from Figure 1, the critical flow velocity of fluid-conveying nanotubes with extra simple support is substantially higher than those without extra support, and the critical flow velocities also rise first and then falls, which is consistent with first-order natural frequency [27].

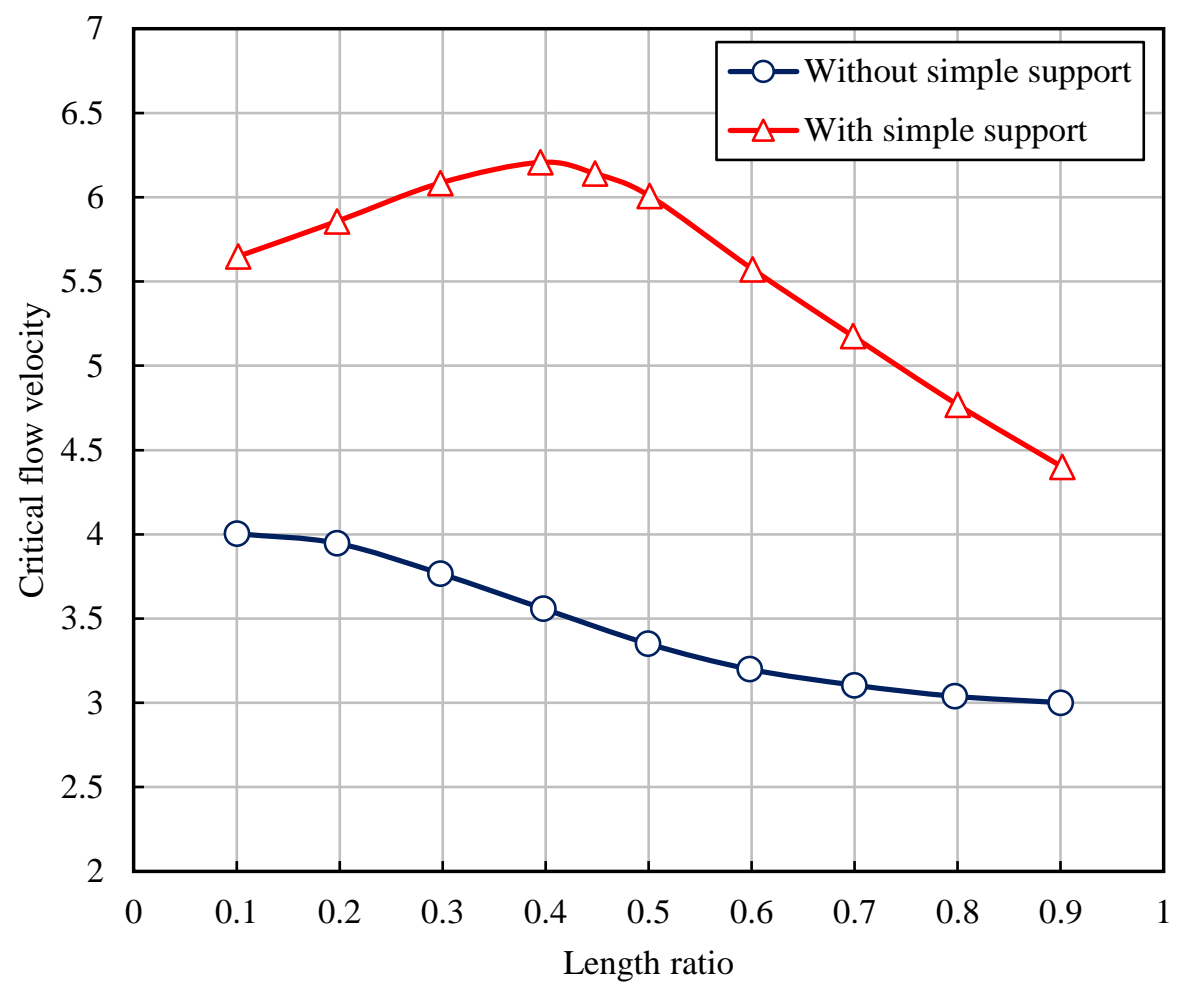

Figure 1: Critical flow velocities in terms of length ratios [27].

Furthermore, Arani et al. [34] investigated the impacts of aspect ratios, Knudson number, and van der Waals forces on the evaluation of the stability of a double-walled boron nitride nanotube conveying fluid by using a nonlocal Timoshenko beam theory. Yan et al. [35] investigated the stability of triplewalled CNTs conveying fluid. They calculated the frequency of the beam and studied the effects of internal flow and van der Waals forces on the stability of carbon nanotubes. Yoon et al. [26] considered the vibration of carbon nanotube conveying fluid. They applied the vibration equation of the nanotube and examined the effects of inner flow on the frequency of carbon nanotubes. Their results suggested that internal moving fluid might have a significant impact on resonance frequency, particularly for suspended longer carbon nanotubes.

\section{SIZE-DEPENDENT VIBRATION}

To analyze micro/nanostructures, theories of continuum mechanics such as classical and size-dependent theories can be applied. Because classical theory does not account for submicron structural discontinuity, it is incapable to capture size-dependent impact as the scale shifts to micro or nano [36]. Size-dependent approaches, such as non-local [37, 38], strain gradient [39], and couple stress [40], are preferable solutions and can provide more accurate results in these situations. It should be noticed that the aforementioned theories contain parameters of size-dependent, the values of precise of which must be validated by experimental data [41] analytical [42, 43], or simulated results [44]. The fabrication of material for conducting simulation or experimental analysis is a simple procedure for basic structures 
including graphene sheets or carbon nanotubes, but for composite structures, the procedure becomes difficult, forcing scientists to approximate the models using mathematics and hypotheses. Many studies have used nonlocal elasticity theory for analyzing features of size-dependent vibration of fluidconveying nanotubes [45-47]. For instance, Liang and Su [45] investigated the effects of nonlocal effects, fluid viscosity, and system factors on the fundamental frequency, critical flow velocity, and dynamic stability of carbon nanotube.

\section{NON-LOCAL STRAIN GRADIENT}

Nonlocal elasticity theory is a higher-order continuum theory than local elasticity theory. As previously stated, local continuum mechanics implies that the stress state at a specific location is completely dependent on the strain state at the same point. Non-local continuum mechanics, on the other hand, considers the stress state at a particular location as a function of the strain states of all points in the body, which reveals the scale effect in elasticity. Therefore, nonlocal continuum mechanics theory describes information regarding long-range forces between atoms, and the internal length scale may be thought of simply as a material parameter in the constitutive equations. The non-local elasticity hypothesis assumes that the stress state at a particular point is a stress state's function at all other points in the body. Hence, the theory considers the impact of small length scales of nanomaterials. Askes and Aifantis [48] have proved that the nonlocal elasticity theory is essentially a stress gradient elasticity theory that can be employed to address static problems. However, in the case of dynamic study of carbon nanotube conveying-fluid, nonlocal beam and shell models may be insufficient options for evaluating such systems' dynamic response. Some articles have been published on the use of strain gradient theory for dynamic investigations of nanotubes [49-52]. For example, Dang et al. [49] developed a model of a functionally graded nanotube conveying fluid embedded in an elastic medium based on nonlocal strain gradient theory. They concluded that as the parameter of material length scale was raised, the non-linear frequencies and critical flow velocities rose. In the study of Thong et al. [50], the vibrational responses of functionally graded carbon nanotube-reinforced composite nano-plates were effectively analyzed using an analytical solution based on non-local strain gradient theory. They demonstrated that the nonlocal parameter and strain gradient coefficient have a substantial influence on natural frequencies, notably for higher vibrational modes and thicker nano-plates. Jin et al. [51] developed a higher-order size-dependent beam model, which is coupled with non-local stress, strain gradient impact, surface energy effect, and free vibration modeling of functionally graded nano-tube. The nanotube conveying fluid has dual impacts on linear and nonlinear frequencies.

Wang [53] used strain gradient elasticity theory to examine the vibration behavior of fluid-carrying nanotubes. Kaviani and Mirdamad [54] analyzed wave propagation in CNTs conveying fluid using continuum mechanics size-dependent strain/inertia gradient theory. In comparison to carbon nanotubes, the literature on the dynamics of BN nanotubes conveying fluid is few. In this regard, it can mention the research done by Arani et al. [55], who considered the nonlinear vibration and instability of doublewalled BN nanotubes using the modified couple stress theories. In addition, Ansari et al. [56] investigated the size-dependent non-linear free vibration and instabilities of fluid-conveying singlewalled boron nitride nanotube (SWBNNT) by using a higher-order continuum model based on the modified strain gradient elastic theories. The size impact was captured using the modified strain gradient theory. To examine the nonlinear influence, geometric non-linearity was introduced to generate non-linear governing equations of motion. In their study, the system under examination, as illustrated in Figure 2, is single-walled boron nitride nanotube modeled as a Timoshenko beam with length L, thickness $h$, inner radius $r_{1}$, outer radius $r_{2}$, that is fluid-conveying and set in a viscoelastic medium. The linear visco-Pasternak foundation model was adapted for simulating the viscoelastic medium.

Ansari et al. [56] determined that as the length scale parameter is big, it has a significant impact on the fluctuation of natural frequency. As a result, the classical continuum model is insufficient to forecast the behavior of nonlinear vibration, and a higher-order continuum model is required to lower the relative inaccuracy. By considering recent works, it was found that more study is required to investigate the 
most outstanding approaches to tackle the dynamic and static issues of boron nitride nanotubes conveying fluid in their practical application.

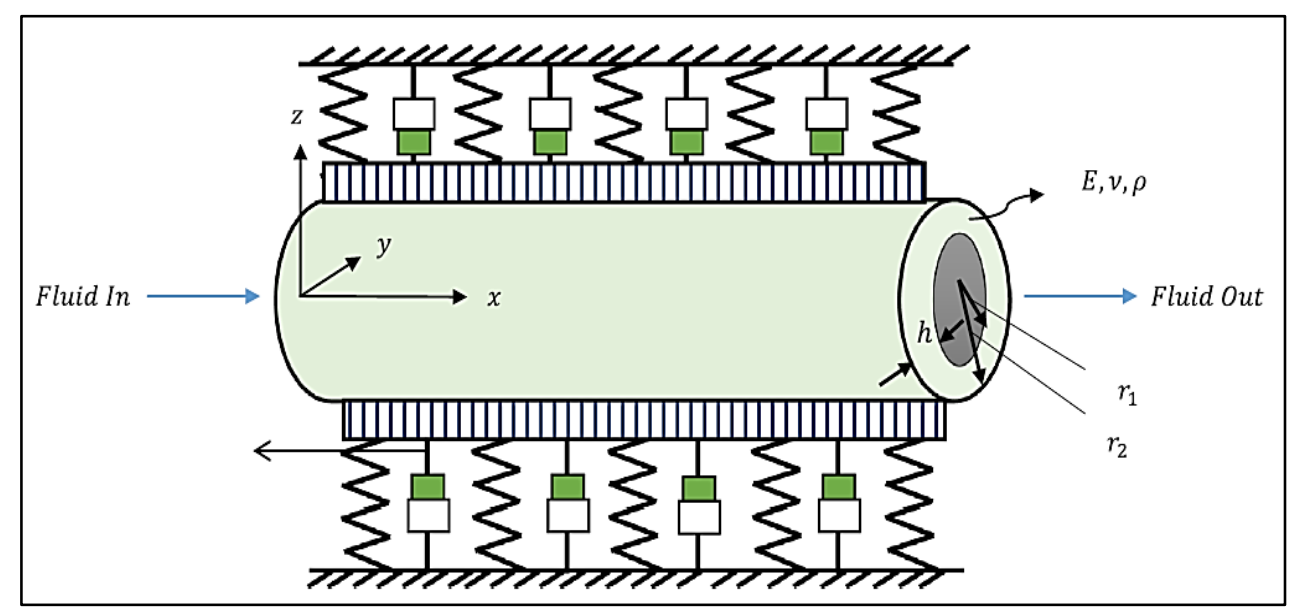

Figure 2: Concept of fluid-conveying SWBNNT [56].

\section{THERMAL VIBRATION OF NANOTUBE}

Carbon nanotubes have been utilized in gas storage, fluid conveying, and drug delivery due to their excellent mechanical, thermal, and electrical characteristics $[57,58]$. Thermal vibration analysis should be performed on fluid-conveying CNTs due to the impact of thermal fluctuations on the mechanical characteristics of nanostructures is required significantly [59]. Multi-wall carbon nanotube (MWCNT) has a wide range of potential applications, including water-proof and tear-resistant cloth fabrics and concrete based on strength, electrical circuits based on electrical conductivity, sensors based on thermal conductivity, and even as a drug delivery vessel [60]. In the study of Malikan et al. [60], the damped vibration of SWCNTs is investigated in this work utilizing a novel shear deformation beam theory. The SWCNT is treated as a flexible beam placed in a viscoelastic foundation and subjected to a transverse dynamic load. The impact of non-local parameters, half-wavelength, damper, temperature, and material changes on the dynamic vibration of nanotubes are thoroughly examined in their research. Figure 3 depicts the effects of in-plane force resultants produced from temperature fluctuations vs many higher and lower-order non-local dimensionless factors in thermal environments.

It can be observed that modifying the heat in the environment raised the difference between outcomes of higher and lower-order non-local situations dramatically. The difference exhibited a rising trend, demonstrating the significance of using higher-order nonlocal strain gradient cases at high temperatures. As a result, at smaller deflection values, there may be no need to adopt the higher-order non-local strain gradient theories [60]. Sedighi et al. [61] simultaneously considered the impacts of length ratio, size dependence, magnetic field, and temperature environment on the nonlinear vibrational properties of composite CNT/BNNT. Figure 4 depicts a doubly clamped nano-heterostructure composed of two different types of tubular materials. The examined composite (hybrid) nanotube's first component is a homogeneous CNT, while the second component is a BNNT. It is considered that two parts have the same geometrical characteristics with various material constants. As it can be seen in Figure 4, the overall length of the hetero-nanotube is $\mathrm{L}$, whereas the lengths of the two segments are $\mathrm{L}_{1}=\xi \mathrm{L}, 0<\xi<1$, and $\mathrm{L}_{2}=(1-\xi) \mathrm{L}$. Using Maxwell's law assumptions, the existence of a magnetic field in space generates a force with a perpendicular direction to the magnetic field. As a result, an axial magnetic field Hx produces a transversal membrane force known as the Lorentz force. It is believed that the entire system is subjected to an axial magnetic field $\mathrm{Hx}$ as well as a thermal environment. Temperature gradients are well known to change the dynamic behavior of nanotubes. The coefficients of thermal expansion of CNT and BNNT, on the other hand, are significantly different, which may 
affect the structural stability of hybrid nanotubes. As a result, the effect of the magneto-thermal environment is also considered while investigating the vibration characteristics of such composite structures. Sedighi et al. [61] demonstrated that when the contribution of the boron-nitride section is dominant, the location of the greatest amplitude would shift to the left when considering larger temperature gradients. In addition, they found that the configurations of the non-linear mode forms are substantially influenced by the high-temperature environment.

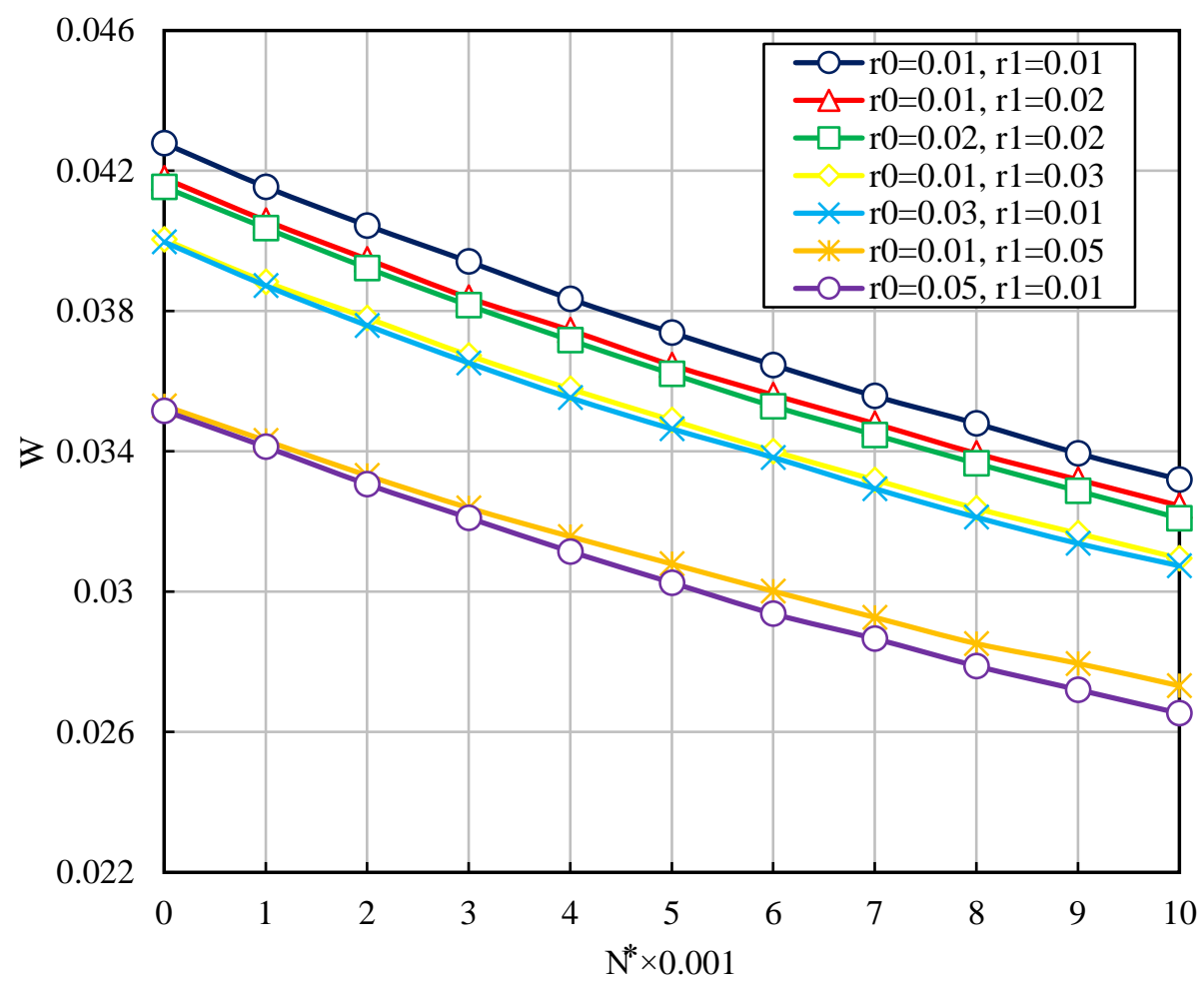

Figure 3: Temperature variations in terms of higher and lower-order non-local factors [60].

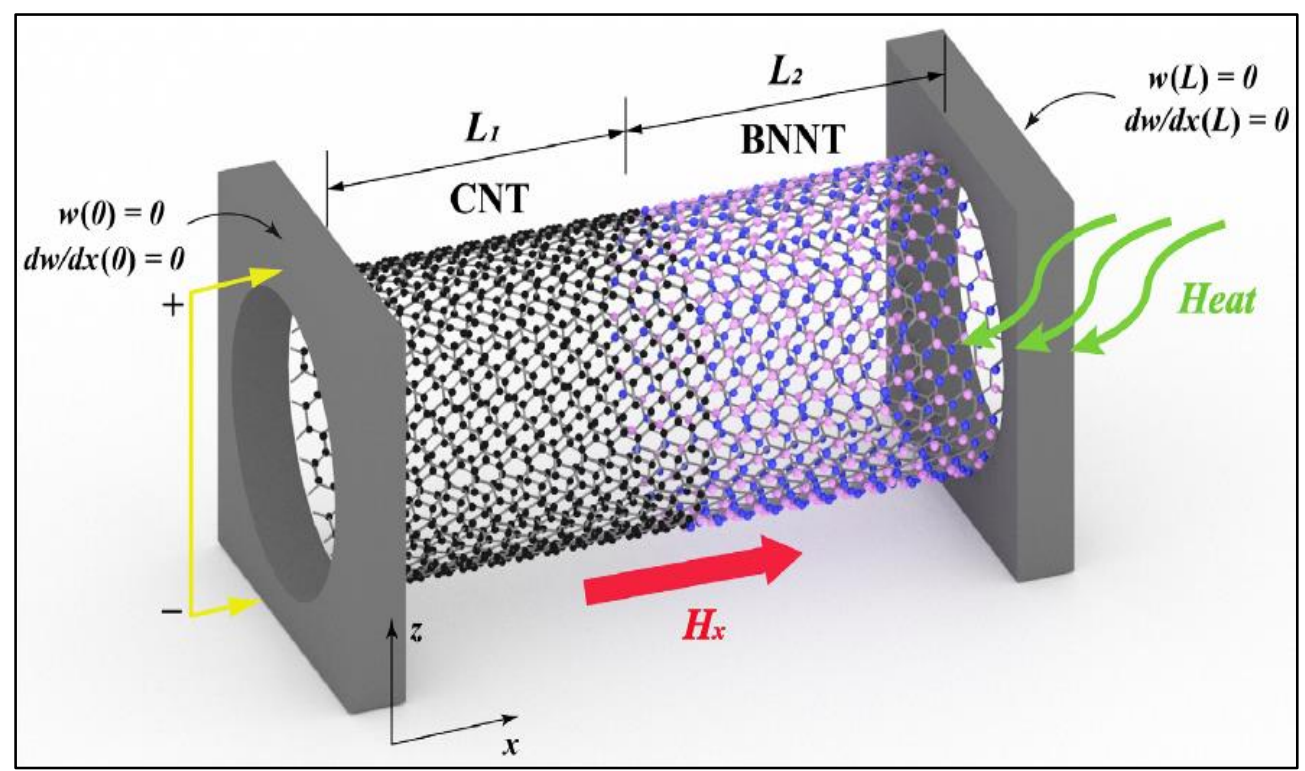

Figure 4: The schematic layout of a C/BN nanotube-based doubly clamped nano-heterostructure [61]. 
Furthermore, Sedighi et al. [62] investigated the impacts of magneto-thermal field, and sizedependence on divergence velocity, and mode shapes of fluid-conveying NTs. It can be concluded that any increase in temperature raises the divergence velocity in a low-temperature media, but any increase in temperature decreases the critical velocity in a high-temperature media.

Quantum effects in nanoscale electronics and structures might become extremely significant as thermal vibration is considered, according to past studies [63-65]. By evaluating the specific heat of a pure SWCNT, Hone et al. [63] verified the quantization of phonon spectra. Using a phenomenological force-constant model, Zimmermann et al. [64] demonstrated that the quantum size impact on CNTs might be seen through phonon thermal conductance. Researchers developed alternative semi-classical techniques [65] based on quantum theory that may be used to investigate the dynamics of nano-systems.

In the study of Zhang et al. [59], a nonlocal elastic model is utilized to consider the effects of quantum on thermal vibration of a fluid-conveying single-walled CNT. Figure 5 demonstrates the RMS amplitude of thermal vibration of fluid-conveying Single-walled CNT at several values of temperatures. They concluded that the Root Mean Squared (RMS) amplitude of thermal vibration of Single-walled CNT conveying fluid anticipated by quantum theory is lower than that anticipated by the law of energy equipartition.

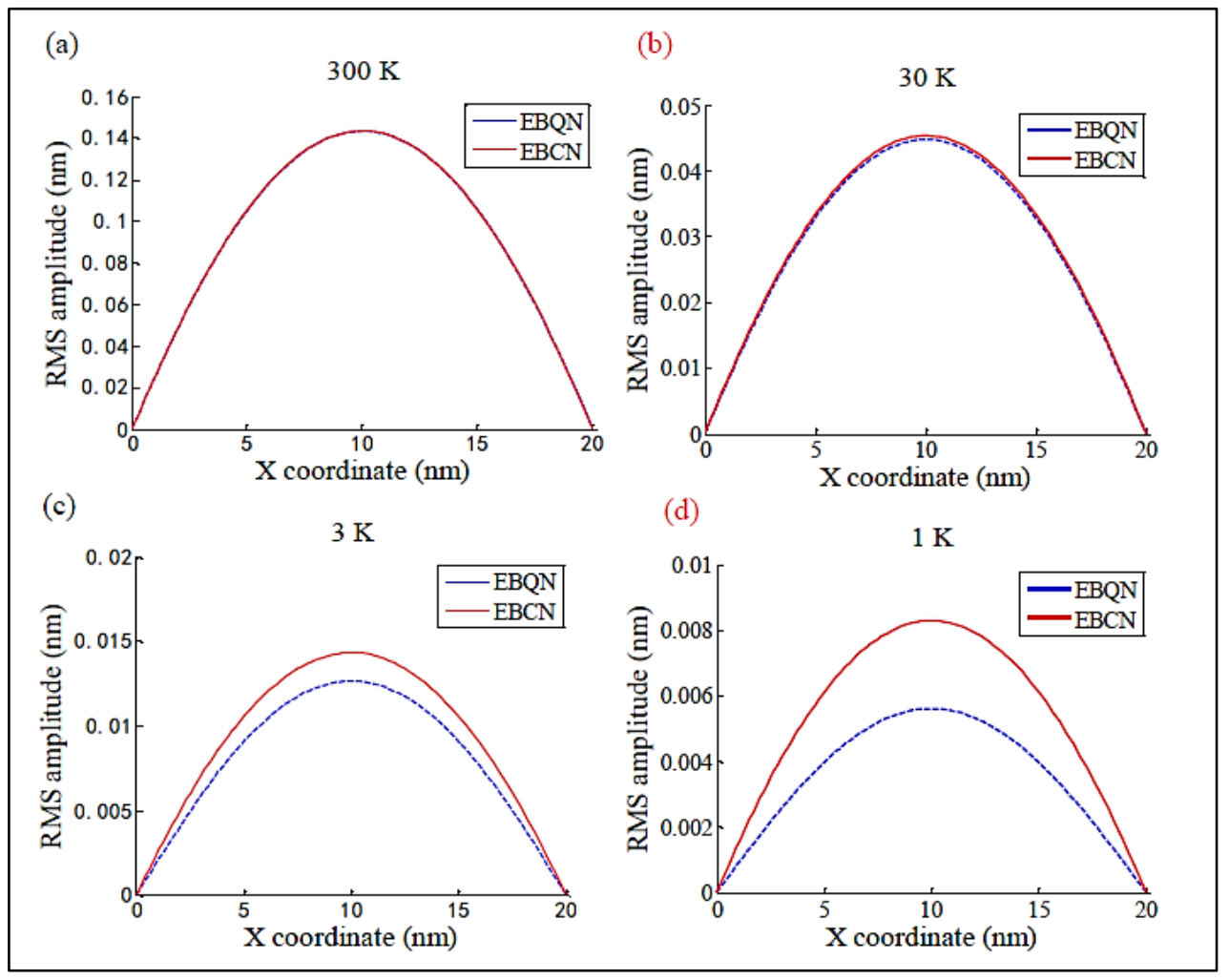

Figure 5: RMS amplitude of thermal vibration of conveying fluid CNT at specific temperatures [59].

Thermal fluctuations are closely connected to the resonance characteristics of low-dimensional structures, which operate as nanoscale devices [66, 67]. The thermal vibration concerns of a carbon nanotube, which may be utilized as a nano-electronic component [68], and an AFM tip [69] have attracted the interest of scientists. The amplitude of the natural thermal vibrations of CNTs is measured using transmission electron microscopy to determine their Young's modulus [70]. Moser et al. [71] investigated carbon nanotube mechanical resonators at cryostat temperatures of $1.2 \mathrm{~K}$ and $30 \mathrm{mK}$, respectively, utilizing an ultrasensitive technique based on cross-correlated electrical noise measurements combined with parametric downconversion. Molecular dynamics (MD) and continuum models [72, 73], in addition to experimental approaches, are critical tools for studying thermal vibration 
in CNTs. Because of its complexity, the pure quantum approach is difficult to represent in full the dynamics of many-body systems [74]. However, several researchers have indicated that when the carbon nanotube vibrates at a low enough temperature, the quantum effects should be addressed [75]. To meet these problems, many semi-classical approaches for incorporating quantum effects into the dynamics of nano-systems have been developed. Wang and $\mathrm{Hu}$ [76] investigated the thermal vibration of a carbon nanotube utilizing classical beam theory and quantum effects instead of the law of energy equipartition. They demonstrated that the quantum theory predicts a smaller RMS amplitude of thermal vibration of a carbon nanotube than the law of energy equipartition. Liu and Wang [74] conducted a thorough investigation of the thermal vibrations of cantilever single-walled carbon nanotubes utilizing molecular dynamics with a quantum heat bath. Figure 6 depicts the RMS amplitude at varying sections of a $9.84 \mathrm{~nm}$ long $(5,5)$ single-walled carbon nanotube at $\mathrm{T}=5 \mathrm{~K}, \mathrm{~T}=3 \mathrm{~K}$, and $\mathrm{T}=1 \mathrm{~K}$. These findings imply that the quantum effect is more relevant for a single-walled carbon nanotube thermal vibration at lower temperatures. Consequently, they concluded that the root of mean squared amplitude of the thermal vibration of a single-walled carbon nanotube obtained from semi-quantum molecular dynamics is lower than that obtained from classical molecular dynamics, particularly at very low temperatures and high-order modes.

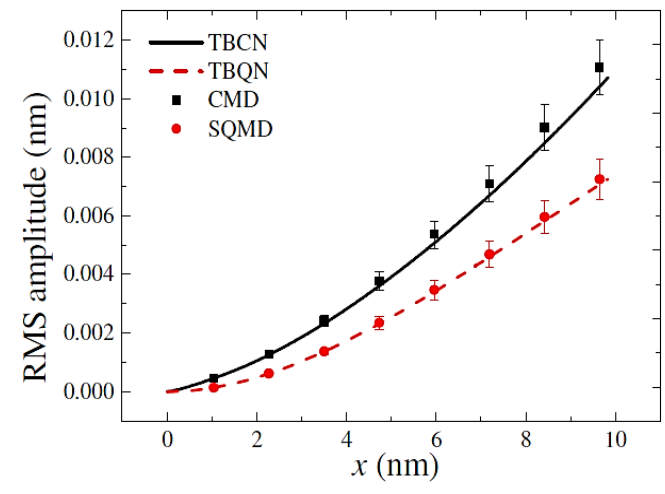

(a)

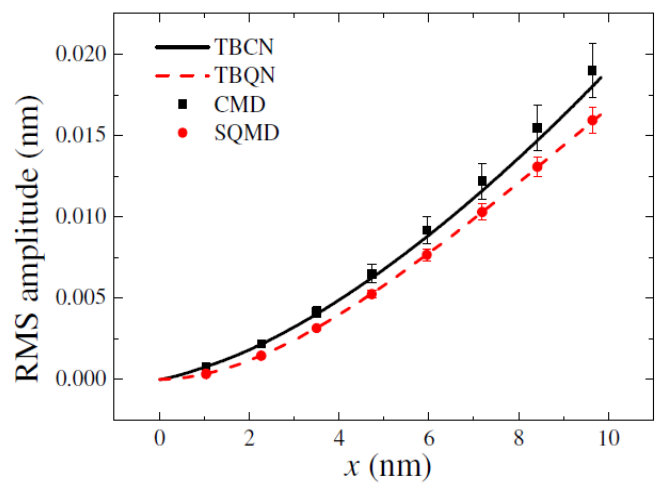

(b)

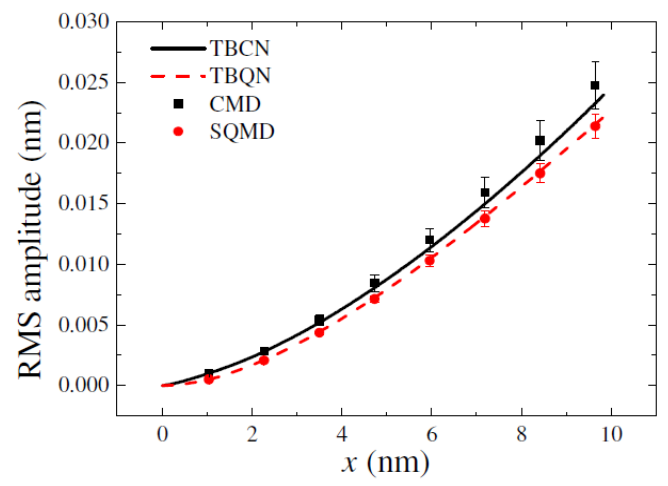

(c)

Figure 6: RMS amplitude at $\mathrm{T}=5 \mathrm{~K}, \mathrm{~T}=3 \mathrm{~K}$ and $\mathrm{T}=1 \mathrm{~K}$ [74] 


\section{CONCLUSION}

The aim of the present review, which is primarily a brief overview of fluid-conveying nanotubes, is to discuss the dynamical characteristics of size-dependent vibration and non-local strain gradient theory of fluid-conveying nanotubes. Furthermore, the few and remarkable works on the vibration of fluidconveying nanotubes in a thermal environment, as well as thermal vibration concerns of carbon nanotubes have been examined and analyzed in depth. By considering significant studies on the vibration of a fluid-conveying nanotube, it was observed that fluid flow inside carbon nanotubes is a crucial and challenging scientific topic. Nanotubes are a key component of modern nanostructures applications due to their unique mechanical, thermal, electrical, and physical properties. For analyzing micro/nanostructures, theories of continuum mechanics namely classical and size-dependent theories can be conducted and it was realized that non-local, strain gradient, and couple stress are size-dependent methodologies that are superior solutions and can deliver results that are more appropriate. Because of the considerable impact of thermal fluctuations on the mechanical characteristics of nanostructures, thermal vibration analysis on fluid-conveying CNTs is necessary. The dynamic behavior of nanotubes is known to be affected by temperature gradients. The coefficients of thermal expansion of carbon and boron nitride nanotubes, on the other hand, are significantly different, which may influence hybrid nanotube structural stability. Thermal fluctuations are intimately connected to the resonance properties of low-dimensional structures that function as nanoscale devices. Furthermore, several studies have revealed that the quantum effects should be addressed as the carbon nanotube vibrates at a low enough temperature.

\section{REFERENCES}

[1] Yan, J. W., He, J. B., \& Tong, L. H. (2019). Longitudinal and torsional vibration characteristics of boron nitride nanotubes. Journal of Vibration Engineering \& Technologies, 7(3), 205-215.

[2] Fatahian, H., Hosseini, E., \& Fatahian, E. (2020). CFD simulation of a novel design of square cyclone with dual-inverse cone. Advanced Powder Technology, 31(4), 1748-1758.

[3] Mahinzare, M., Mohammadi, K., \& Ghadiri, M. (2021). A nonlocal strain gradient theory for vibration and flutter instability analysis in rotary SWCNT with conveying viscous fluid. Waves in Random and Complex Media, 31(2), 305-330.

[4] Fatahian, E., Fatahian, H., Hosseini, E., \& Ahmadi, G. (2021). A low-cost solution for the collection of fine particles in square cyclone: A numerical analysis. Powder Technology, 387, 454-465.

[5] Fatahian, H., Salarian, H., Nimvari, M. E., \& Fatahian, E. (2018). Numerical study of thermal characteristics of fuel oil-alumina and water-alumina nanofluids flow in a channel in the laminar flow. IIUM Engineering Journal, 19(1), 251-269.

[6] Fatahian, E., Salarian, H., \& Fatahian, H. (2020). A parametric study of the heat exchanger copper coils used in an indirect evaporative cooling system. SN Applied Sciences, 2(1), 1-10.

[7] Fatahian, R., Mirjalili, M., Khajavi, R., Rahimi, M. K., \& Nasirizadeh, N. (2021). Effect of electrospinning parameters on production of polyvinyl alcohol/polylactic acid nanofiber using a mutual solvent. Polymers and Polymer Composites, 09673911211027126.

[8] Fatahian, R., Mirjalili, M., Khajavi, R., Rahimi, M. K., \& Nasirizadeh, N. (2020). A novel hemostat and antibacterial nanofibrous scaffold based on poly (vinyl alcohol)/poly (lactic acid). Journal of Bioactive and Compatible Polymers, 35(3), 189-202.

[9] Fatahian, R., Mirjalili, M., Khajavi, R., Rahimi, M. K., \& Nasirizadeh, N. (2020). Fabrication of antibacterial and hemostatic electrospun PVA nanofibers for wound healing. SN Applied Sciences, 2(7), 1-7.

[10] Chiu, H. Y., Hung, P., \& Postma, H. W. (2008). Ch. \& Bockrath, M. Atomic-scale mass sensing using carbon nanotube resonators. Nano Lett, 8(12), 4342-4346. 
[11] Bunch, J. S., Van Der Zande, A. M., Verbridge, S. S., Frank, I. W., Tanenbaum, D. M., Parpia, J. M., ... \& McEuen, P. L. (2007). Electromechanical resonators from graphene sheets. Science, 315(5811), 490-493.

[12] Randjbaran, E., Majid, D. L., Zahari, R., Sultan, M. T., \& Mazlan, N. (2020). Effects of volume of carbon nanotubes on the angled ballistic impact for carbon kevlar hybrid fabrics. Facta Universitatis, Series: Mechanical Engineering, 18(2), 229-244.

[13] Rysaeva, L. K., Bachurin, D. V., Murzaev, R. T., Abdullina, D. U., Korznikova, E. A., Mulyukov, R. R., \& Dmitriev, S. V. (2020). Evolution of the carbon nanotube bundle structure under biaxial and shear strains. Facta Universitatis, Series: Mechanical Engineering, 18(4), 525536.

[14] Pujadó, M. P. (2012). Carbon nanotubes as platforms for biosensors with electrochemical and electronic transduction. Springer Science \& Business Media.

[15] Liu, F., Wagterveld, R. M., Gebben, B., Otto, M. J., Biesheuvel, P. M., \& Hamelers, H. V. M. (2014). Carbon nanotube yarns as strong flexible conductive capacitive electrodes. Colloid and Interface Science Communications, 3, 9-12.

[16] Parker, C. B., Raut, A. S., Brown, B., Stoner, B. R., \& Glass, J. T. (2012). Three-dimensional arrays of graphenated carbon nanotubes. Journal of Materials Research, 27(7), 1046.

[17] Pakdel, A., Zhi, C., Bando, Y., \& Golberg, D. (2012). Low-dimensional boron nitride nanomaterials. Materials Today, 15(6), 256-265.

[18] Zhi, C., Bando, Y., Tang, C., \& Golberg, D. (2010). Boron nitride nanotubes. Materials Science and Engineering: R: Reports, 70(3-6), 92-111.

[19] Mojahedi, M. (2017). Size dependent dynamic behavior of electrostatically actuated microbridges. International Journal of Engineering Science, 111, 74-85.

[20] Qi, L., Huang, S., Fu, G., Zhou, S., \& Jiang, X. (2018). On the mechanics of curved flexoelectric microbeams. International Journal of Engineering Science, 124, 1-15.

[21] Farokhi, H., \& Ghayesh, M. H. (2018). Supercritical nonlinear parametric dynamics of Timoshenko microbeams. Communications in Nonlinear Science and Numerical Simulation, 59, 592-605.

[22] Farajpour, A., Farokhi, H., Ghayesh, M. H., \& Hussain, S. (2018). Nonlinear mechanics of nanotubes conveying fluid. International Journal of Engineering Science, 133, 132-143.

[23] Ghorbani, K., Rajabpour, A., \& Ghadiri, M. (2021). Determination of carbon nanotubes sizedependent parameters: Molecular dynamics simulation and nonlocal strain gradient continuum shell model. Mechanics Based Design of Structures and Machines, 49(1), 103-120.

[24] Sokhan, V. P., Nicholson, D., \& Quirke, N. (2002). Fluid flow in nanopores: Accurate boundary conditions for carbon nanotubes. The Journal of chemical physics, 117(18), 8531-8539.

[25] Al-Furjan, M. S. H., Bolandi, S. Y., Habibi, M., Ebrahimi, F., Chen, G., \& Safarpour, H. (2021). Enhancing vibration performance of a spinning smart nanocomposite reinforced microstructure conveying fluid flow. Engineering with Computers, 1-16.

[26] Yoon, J., Ru, C. Q., \& Mioduchowski, A. (2005). Vibration and instability of carbon nanotubes conveying fluid. Composites Science and Technology, 65(9), 1326-1336.

[27] Cheng, Q., Liu, Y., Wang, G., Liu, H., Jin, M., \& Li, R. (2019). Free vibration of a fluidconveying nanotube constructed by carbon nanotube and boron nitride nanotube. Physica E: Low-dimensional Systems and Nanostructures, 109, 183-190.

[28] Li, J., Furuta, T., Goto, H., Ohashi, T., Fujiwara, Y., \& Yip, S. (2003). Theoretical evaluation of hydrogen storage capacity in pure carbon nanostructures. The Journal of Chemical Physics, 119(4), 2376-2385.

[29] Wang, L. (2009). Dynamical behaviors of double-walled carbon nanotubes conveying fluid accounting for the role of small length scale. Computational Materials Science, 45(2), 584-588.

[30] Zhang, Y. W., Yang, T. Z., Zang, J., \& Fang, B. (2013). Terahertz wave propagation in a nanotube conveying fluid taking into account surface effect. Materials, 6(6), 2393-2399. 
[31] Lee, H. L., \& Chang, W. J. (2009). Vibration analysis of a viscous-fluid-conveying singlewalled carbon nanotube embedded in an elastic medium. Physica E: Low-dimensional Systems and Nanostructures, 41(4), 529-532.

[32] Mirramezani, M., \& Mirdamadi, H. R. (2012). Effects of nonlocal elasticity and Knudsen number on fluid-structure interaction in carbon nanotube conveying fluid. Physica E: Lowdimensional Systems and Nanostructures, 44(10), 2005-2015.

[33] Zeighampour, H., Beni, Y. T., \& Karimipour, I. (2017). Wave propagation in double-walled carbon nanotube conveying fluid considering slip boundary condition and shell model based on nonlocal strain gradient theory. Microfluidics and Nanofluidics, 21(5), 85-100.

[34] Ghorbanpour Arani, A., Hashemian, M., \& Kolahchi, R. (2015). Nonlocal Timoshenko beam model for dynamic stability of double-walled boron nitride nanotubes conveying nanoflow. Proceedings of the Institution of Mechanical Engineers, Part N: Journal of Nanoengineering and Nanosystems, 229(1), 2-16.

[35] Yan, Y., He, X. Q., Zhang, L. X., \& Wang, C. M. (2009). Dynamic behavior of triple-walled carbon nanotubes conveying fluid. Journal of Sound and Vibration, 319(3-5), 1003-1018.

[36] Safarpour, H., Ghanizadeh, S. A., \& Habibi, M. (2018). Wave propagation characteristics of a cylindrical laminated composite nanoshell in thermal environment based on the nonlocal strain gradient theory. The European Physical Journal Plus, 133(12), 532.

[37] Lee, H. L., \& Chang, W. J. (2008). Free transverse vibration of the fluid-conveying singlewalled carbon nanotube using nonlocal elastic theory. Journal of Applied Physics, 103(2), 024302 .

[38] Zenkour, A. M. (2018). A novel mixed nonlocal elasticity theory for thermoelastic vibration of nanoplates. Composite Structures, 185, 821-833.

[39] Mindlin, R. D. (1965). Second gradient of strain and surface tension in linear elasticity. International Journal of Solids and Structures, 1(4), 417-438.

[40] SafarPour, H., Mohammadi, K., Ghadiri, M., \& Rajabpour, A. (2017). Influence of various temperature distributions on critical speed and vibrational characteristics of rotating cylindrical microshells with modified lengthscale parameter. The European Physical Journal Plus, 132(6), $1-19$.

[41] Xiao, S., \& Hou, W. (2006). Studies of size effects on carbon nanotubes' mechanical properties by using different potential functions. Fullerenes, Nanotubes, and Carbon Nonstructures, 14(1), 9-16.

[42] Mohammadimehr, M., Emdadi, M., Afshari, H., \& Rousta Navi, B. (2018). Bending, buckling and vibration analyses of MSGT microcomposite circular-annular sandwich plate under hydrothermo-magneto-mechanical loadings using DQM. International Journal of Smart and Nano Materials, 9(4), 233-260.

[43] Jiang, L., \& Guo, W. (2011). A molecular mechanics study on size-dependent elastic properties of single-walled boron nitride nanotubes. Journal of the Mechanics and Physics of Solids, 59(6), 1204-1213.

[44] Mohammadi, K., Mahinzare, M., Rajabpour, A., \& Ghadiri, M. (2017). Comparison of modeling a conical nanotube resting on the Winkler elastic foundation based on the modified couple stress theory and molecular dynamics simulation. The European Physical Journal Plus, 132(3), 1-18.

[45] Liang, F., \& Su, Y. (2013). Stability analysis of a single-walled carbon nanotube conveying pulsating and viscous fluid with nonlocal effect. Applied Mathematical Modelling, 37(10-11), 6821-6828.

[46] Ansari, R., \& Ramezannezhad, H. (2011). Nonlocal Timoshenko beam model for the largeamplitude vibrations of embedded multiwalled carbon nanotubes including thermal effects. Physica E: Low-dimensional Systems and Nanostructures, 43(6), 1171-1178. 
[47] Soltani, P., \& Farshidianfar, A. (2012). Periodic solution for nonlinear vibration of a fluidconveying carbon nanotube, based on the nonlocal continuum theory by energy balance method. Applied Mathematical Modelling, 36(8), 3712-3724.

[48] Askes, H., \& Aifantis, E. C. (2009). Gradient elasticity and flexural wave dispersion in carbon nanotubes. Physical Review B, 80(19), 195412.

[49] Dang, V. H., Sedighi, H. M., Civalek, O., \& Abouelregal, A. E. (2021). Nonlinear vibration and stability of FG nanotubes conveying fluid via nonlocal strain gradient theory. Structural Engineering and Mechanics, 78(1), 103-116.

[50] Thang, P. T., Tran, P., \& Nguyen-Thoi, T. (2021). Applying nonlocal strain gradient theory to size-dependent analysis of functionally graded carbon nanotube-reinforced composite nanoplates. Applied Mathematical Modelling, 93, 775-791.

[51] Jin, Q., Ren, Y., Jiang, H., \& Li, L. (2021). A higher-order size-dependent beam model for nonlinear mechanics of fluid-conveying FG nanotubes incorporating surface energy. Composite Structures, 114022.

[52] Ghazavi, M. R., \& Molki, H. (2018). Nonlinear analysis of the micro/nanotube conveying fluid based on second strain gradient theory. Applied Mathematical Modelling, 60, 77-93.

[53] Wang, L. (2012). Vibration analysis of nanotubes conveying fluid based on gradient elasticity theory. Journal of Vibration and Control, 18(2), 313-320.

[54] Kaviani, F., \& Mirdamadi, H. R. (2013). Wave propagation analysis of carbon nano-tube conveying fluid including slip boundary condition and strain/inertial gradient theory. Computers \& Structures, 116, 75-87.

[55] Arani, A. G., Bagheri, M. R., Kolahchi, R., \& Maraghi, Z. K. (2013). Nonlinear vibration and instability of fluid-conveying DWBNNT embedded in a visco-Pasternak medium using modified couple stress theory. Journal of Mechanical Science and Technology, 27(9), 26452658.

[56] Ansari, R., Norouzzadeh, A., Gholami, R., Shojaei, M. F., \& Hosseinzadeh, M. (2014). Sizedependent nonlinear vibration and instability of embedded fluid-conveying SWBNNTs in thermal environment. Physica E: Low-dimensional Systems and Nanostructures, 61, 148-157.

[57] Cai, D., Mataraza, J. M., Qin, Z. H., Huang, Z., Huang, J., Chiles, T. C., \& Ren, Z. (2005). Highly efficient molecular delivery into mammalian cells using carbon nanotube spearing. Nature Methods, 2(6), 449-454.

[58] Pastorin, G., Wu, W., Wieckowski, S., Briand, J. P., Kostarelos, K., Prato, M., \& Bianco, A. (2006). Double functionalisation of carbon nanotubes for multimodal drug delivery. Chemical communications, (11), 1182-1184.

[59] Zhang, Y. W., Zhou, L., Fang, B., \& Yang, T. Z. (2017). Quantum effects on thermal vibration of single-walled carbon nanotubes conveying fluid. Acta Mechanica Solida Sinica, 30(5), 550556.

[60] Malikan, M., Nguyen, V. B., \& Tornabene, F. (2018). Damped forced vibration analysis of single-walled carbon nanotubes resting on viscoelastic foundation in thermal environment using nonlocal strain gradient theory. Engineering Science and Technology, an International Journal, 21(4), 778-786.

[61] Sedighi, H. M., Malikan, M., Valipour, A., \& Żur, K. K. (2020). Nonlocal vibration of carbon/boron-nitride nano-hetero-structure in thermal and magnetic fields by means of nonlinear finite element method. Journal of Computational Design and Engineering, 7(5), 591602.

[62] Sedighi, H. M., Ouakad, H. M., Dimitri, R., \& Tornabene, F. (2020). Stress-driven nonlocal elasticity for the instability analysis of fluid-conveying C-BN hybrid-nanotube in a magnetothermal environment. Physica Scripta, 95(6), 065204.

[63] Hone, J., Batlogg, B., Benes, Z., Johnson, A. T., \& Fischer, J. E. (2000). Quantized phonon spectrum of single-wall carbon nanotubes. Science, 289(5485), 1730-1733. 
[64] Zimmermann, J., Pavone, P., \& Cuniberti, G. (2008). Vibrational modes and low-temperature thermal properties of graphene and carbon nanotubes: Minimal force-constant model. Physical Review B, 78(4), 045410.

[65] Li, C., \& Chou, T. W. (2005). Quantized molecular structural mechanics modeling for studying the specific heat of single-walled carbon nanotubes. Physical Review B, 71(7), 075409.

[66] Garcia-Sanchez, D., San Paulo, A., Esplandiu, M. J., Perez-Murano, F., Forró, L., Aguasca, A., $\&$ Bachtold, A. (2007). Mechanical detection of carbon nanotube resonator vibrations. Physical review letters, 99(8), 085501.

[67] Wei, X. L., Liu, Y., Chen, Q., Wang, M. S., \& Peng, L. M. (2008). The Very-Low Shear Modulus of Multi-Walled Carbon Nanotubes Determined Simultaneously with the Axial Young's Modulus via in situ Experiments. Advanced Functional Materials, 18(10), 1555-1562.

[68] Ahlskog, M., Hakonen, P., Paalanen, M., Roschier, L., \& Tarkiainen, R. (2001). Multiwalled carbon nanotubes as building blocks in nanoelectronics. Journal of low temperature physics, 124(1), 335-352.

[69] Ishikawa, M., Yoshimura, M., \& Ueda, K. (2002). A study of friction by carbon nanotube tip. Applied Surface Science, 188(3-4), 456-459.

[70] Krishnan, A., Dujardin, E., Ebbesen, T. W., Yianilos, P. N., \& Treacy, M. M. J. (1998). Young's modulus of single-walled nanotubes. Physical review B, 58(20), 14013.

[71] Moser, J., Eichler, A., Güttinger, J., Dykman, M. I., \& Bachtold, A. (2014). Nanotube mechanical resonators with quality factors of up to 5 million. Nature nanotechnology, 9(12), 1007-1011.

[72] Barnard, A. W., Sazonova, V., Van Der Zande, A. M., \& McEuen, P. L. (2012). Fluctuation broadening in carbon nanotube resonators. Proceedings of the National Academy of Sciences, 109(47), 19093-19096.

[73] Wang, L. F., \& Hu, H. Y. (2012). Thermal vibration of double-walled carbon nanotubes predicted via double-Euler-beam model and molecular dynamics. Acta Mechanica, 223(10), 2107-2115.

[74] Liu, R., \& Wang, L. (2015). Thermal vibration of a single-walled carbon nanotube predicted by semiquantum molecular dynamics. Physical Chemistry Chemical Physics, 17(7), 5194-5201.

[75] Hone, J., Batlogg, B., Benes, Z., Johnson, A. T., \& Fischer, J. E. (2000). Quantized phonon spectrum of single-wall carbon nanotubes. Science, 289(5485), 1730-1733.

[76] Wang, L., \& Hu, H. (2014). Thermal vibration of single-walled carbon nanotubes with quantum effects. Proceedings of the Royal Society A: Mathematical, Physical and Engineering Sciences, 470(2168), 20140087. 